\title{
ALMOST CLOSED 1-FORMS
}

\author{
R. PANDHARIPANDE \\ Department of Mathematics, ETH Zürich, Zurich, Switzerland \\ e-mail:rahul@math.ethz.ch \\ and R. P. THOMAS \\ Department of Mathematics, Imperial College, London, SW7 2AZ, United Kingdom \\ e-mail: richard.thomas@imperial.ac.uk
}

(Received 3 July 2012; accepted 22 January 2013; first published online 13 August 2013)

\begin{abstract}
We construct an algebraic almost closed 1-form with zero scheme not expressible (even locally) as the critical locus of a holomorphic function on a nonsingular variety. The result answers a question of Behrend-Fantechi. We correct here an error in our paper (D. Maulik, R Pandharipande and R. P. Thomas, Curves on K3 surfaces and modular forms, J. Topol. 3 (2010) 937-996. arXiv:1001.2719v3), where an incorrect construction with the same claimed properties was proposed.
\end{abstract}

2010 Mathematics Subject Classification. 13D10, 14N35, 14J32.

1. Introduction. An algebraic 1-form $\omega$ on a non-singular variety $V$ is almost closed if $d \omega$ vanishes on the zero scheme $\mathcal{Z}(\omega) \subset V$ of $\omega$. For example, for every non-constant polynomial $F \in \mathbb{C}[x, y]$, the 1 -form

$$
\omega=d F+F d z
$$

is almost closed, but not closed, on $\mathbb{C}^{3}$ with coordinates $x, y, z$. By a construction of Behrend and Fantechi [2], the zero scheme $\mathcal{Z}(\omega)$ carries a natural symmetric obstruction theory in case $\omega$ is almost closed.

Following is the question asked by Behrend and Fantechi [1, 2]: If $\omega$ is almost closed, can we find an analytic neighbourhood

$$
\mathcal{Z} \subset U \subset V
$$

and a holomorphic function $\Phi$ on $U$ so that the equality

$$
\mathcal{Z}(\omega)=\mathcal{Z}(d \Phi)
$$

holds as subschemes? In other words, is $\mathcal{Z}(\omega)$ always the critical locus of a holomorphic function? In example (1), the function

$$
\Phi=e^{z} F
$$

provides an affirmative answer. Such a $\Phi$ is called a potential for $\mathcal{Z}$.

We will construct a non-reduced point at the origin

$$
\mathcal{Z} \subset \mathbb{C}^{2}
$$


which is the zero scheme of an algebraic almost closed 1-form on a Zariski open neighbourhood of the orgin in $\mathbb{C}^{2}$, but not the critical locus of any holomorphic function defined in a neighbourhood of the origin in $\mathbb{C}^{2}$. We also show that $\mathcal{Z}$ cannot be a critical locus of a holomorphic function defined locally on $\mathbb{C}^{n}$ for any $n$.

We will primarily study almost closed 1-forms via formal power series analysis at the origin. In Section 4 we show how these methods also yield algebraic almost closed 1-forms on Zariski open neighbourhoods of the origin as relevant to the BehrendFantechi question.

The context of the problem that this paper addresses is Donaldson-Thomas theory and its generalisations. One tries to define invariants of Calabi-Yau 3-fold $X$ by virtual counting of (semi-stable) objects of the derived category of coherent sheaves on $X$. The obstruction theory of such objects is symmetric, so by Behrend [1] the moduli space is locally described as the zero locus of an almost closed 1-form. It is expected that these moduli spaces are in fact locally critical loci of holomorphic functions, and the example of this paper shows that this is a stronger condition than symmetry of the obstruction theory.

Recently, Pantev, Toën, Vaquie and Vezzosi [6] have introduced the notion of a $(-1)$-shifted symplectic structure on a derived scheme. This is a stronger condition than the existence of a symmetric obstruction theory on the underlying scheme $\mathcal{Z}$. They also show that the moduli space of objects in the derived category of a Calabi-Yau 3-fold admits such a structure; and Brav, Bussi and Joyce [3] have proved that a scheme $\mathcal{Z}$ is locally a critical locus if and only if it underlies a derived scheme with (-1)-shifted symplectic structure. In particular, our example cannot carry a (-1)-shifted symplectic structure.

2. Construction of the almost closed 1-form. Consider $\mathbb{C}^{2}$ with coordinates $x$ and $y$. We will use capital letters to denote elements of the ring $\mathbb{C} \llbracket x, y \rrbracket$. In case the element is polynomial and homogenous, the degree is designated in the subscript. As usual partial derivatives will also be denoted by subscripts, so

$$
A_{d, x y}=\frac{\partial^{2} A_{d}}{\partial x \partial y}
$$

is a homogeneous polynomial of degree $d-2$.

We start by constructing an almost closed 1-form $\sigma$ on $\mathbb{C}^{2}$ via formal power series. The construction depends upon the initial choice of a sufficiently large degree $d$. Let

$$
\begin{aligned}
\sigma & =A d x+B d y \\
& =\left(A_{d}+A_{d+1}+A_{d+2}+\cdots\right) d x+\left(B_{d}+B_{d+1}+B_{d+2}+\cdots\right) d y
\end{aligned}
$$

be a 1 -form starting at order $d$ satisfying

$$
\begin{aligned}
A_{y}-B_{x}= & C A+D B=\left(C_{0}+C_{1}+C_{2}+\cdots\right)\left(A_{d}+A_{d+1}+A_{d+2}+\cdots\right) \\
& +\left(D_{0}+D_{1}+D_{2}+\cdots\right)\left(B_{d}+B_{d+1}+B_{d+2}+\cdots\right),
\end{aligned}
$$

where $C, D \in \mathbb{C} \llbracket x, y \rrbracket$. 
The zero scheme $\mathcal{Z}(\sigma)$ is $\operatorname{Spec} \frac{\mathbb{C} \llbracket x, y \rrbracket}{(A, B)}$. We shall often use the trivialisation $d y \wedge d x$ of the 2 -form on $\mathbb{C}^{2}$ to identify $d \sigma$ with the function $A_{y}-B_{x}$. Equation (3) is the almost closed condition,

$$
d \sigma=A_{y}-B_{x} \in(A, B) .
$$

For sufficient large $d$, we will prove that the general $\sigma$ satisfying (3) has zero scheme which is not the critical locus of a holomorphic function.

Fixing $C, D \in \mathbb{C} \llbracket x, y \rrbracket$, we construct solutions $\sigma$ to the almost closed equation (3) by the following procedure:

- First, $A_{d}$ and $B_{d}$ are degree $d$ homogeneous polynomials satisfying

$$
A_{d, y}-B_{d, x}=0
$$

Thus,

$$
A_{d}=P_{d+1, x}, \quad B_{d}=P_{d+1, y},
$$

for any homogeneous degree $d+1$ polynomial $P_{d+1}$. We pick a general solution: a general element of the vector space

$$
\operatorname{ker}\left(\operatorname{Sym}^{d}\left(\mathbb{C}^{2}\right)^{*} \oplus \operatorname{Sym}^{d}\left(\mathbb{C}^{2}\right)^{*} \stackrel{\partial_{y} \oplus-\partial_{x}}{\longrightarrow} \operatorname{Sym}^{d-1}\left(\mathbb{C}^{2}\right)^{*}\right) .
$$

- Next, $A_{d+1}$ and $B_{d+1}$ are homogeneous polynomials of degree $d+1$ satisfying

$$
A_{d+1, y}-B_{d+1, x}=C_{0} A_{d}+D_{0} B_{d}
$$

for the given constants $C_{0}$ and $D_{0}$. The above is an affine linear equation on the vector space of pairs $\left(A_{d+1}, B_{d+1}\right)$ with a non-empty set of solutions (for example, set $B_{d+1}=0$ and integrate to get $A_{d+1}$ ). We pick a general element of the affine space of solutions.

- At the $k$ th order, we have defined $A_{d}, \ldots, A_{k-1}$ and $B_{d}, \ldots, B_{k-1}$, and we pick degree $k$ homogeneous polynomials $A_{k}, B_{k}$ satisfying the affine linear equation

$$
A_{k, y}-B_{k, x}=\sum_{i=0}^{k-1-d} C_{i} A_{k-1-i}+\sum_{i=0}^{k-1-d} D_{i} B_{k-1-i}
$$

Again, the affine space of solutions is non-empty, and we pick a general element.

Thus, we obtain a general formal power series almost-closed 1-form $\sigma$ starting at order $d$ with given $C, D \in \mathbb{C} \llbracket x, y \rrbracket$. Later, we will show $\sigma$ can be modified to be algebraic.

3. Convergence. Although not necessary for our construction, the power series solution $\sigma$ can be taken to be convergent when conditions ${ }^{1}$ are placed on the series $C, D \in \mathbb{C} \llbracket x, y \rrbracket$.

${ }^{1}$ Stronger results are certainly possible, but the conditions we impose are sufficiently mild that the nonexistence result of Theorem 3 still holds if we replace the word 'general' with 'general within the Euclidean open set of Lemma 1'. 
Lemma 1. Suppose $C_{\geq 2}=0=D_{\geq 2}$. Then there is a power series solution $\sigma$ starting at order $d$ with non-zero radius of convergence. Moreover, the set of solutions of (3) which converge contains an Euclidean open set in the space of all solutions.

Proof. When $C_{\geq 2}=0=D_{\geq 2}$, equation (7) simplifies to

$$
A_{k, y}-B_{k, x}=C_{0} A_{k-1}+D_{0} B_{k-1}+C_{1} A_{k-2}+D_{1} B_{k-2} .
$$

Let $\|p\|$ denote the maximum of the absolute values of the coefficients of the polynomial $p(x, y)$, and make the following definitions:

$$
\begin{aligned}
\lambda & =\max \left(\left|C_{0}\right|,\left|D_{0}\right|, 2\left\|C_{1}\right\|, 2\left\|D_{1}\right\|\right), \\
\mu_{k} & =\max \left(\left\|A_{k}\right\|,\left\|B_{k}\right\|,\left\|A_{k-1}\right\|,\left\|B_{k-1}\right\|\right), \\
E_{k-1} & =C_{0} A_{k-1}+D_{0} B_{k-1}+C_{1} A_{k-2}+D_{1} B_{k-2} .
\end{aligned}
$$

We easily find

$$
\begin{aligned}
\left\|E_{k-1}\right\| & \leq\left|C_{0}\right|\left\|A_{k-1}\right\|+\left|D_{0}\right|\left\|B_{k-1}\right\|+2\left\|C_{1}\right\|\left\|A_{k-2}\right\|+2\left\|D_{1}\right\|\left\|B_{k-2}\right\| \\
& \leq 4 \lambda \mu_{k-1} .
\end{aligned}
$$

A general solution of (8) is provided by integrating

$$
\begin{aligned}
& A_{k, y}=F_{k-1}, \\
& B_{k, x}=F_{k-1}-E_{k-1},
\end{aligned}
$$

for a general choice of homogeneous degree $k-1$ polynomial $F_{k-1}$. We now choose $F_{k-1}$ to satisfy

$$
\left\|F_{k-1}\right\|<4 \lambda \mu_{k-1},
$$

which is a Euclidean open condition.

Since integration divides all coefficients by integers, we see

$$
\left\|A_{k}\right\|<4 \lambda \mu_{k-1}, \quad\left\|B_{k}\right\|<8 \lambda \mu_{k-1},
$$

so

$$
\mu_{k} \leq \max (1,8 \lambda) \mu_{k-1} .
$$

Therefore,

$$
\mu_{k} \leq \alpha \beta^{k}
$$

for some constants $\alpha, \beta$. The 1-form $\sigma$ solving (3) thus has radius of convergence at least $\beta^{-1}$.

4. Algebraic almost closed 1-forms. We return to the formal power series solutions $\sigma$ of (3) starting at degree $d$. For general choices of $A_{d}$ and $B_{d}$, the zero locus $\mathcal{Z}(\sigma)$ will be a non-reduced scheme $\mathcal{Z}_{0}(\sigma)$ at the origin of $\mathbb{C}^{2}$ (plus possibly some other disjoint loci). For sufficiently large $N$,

$$
\mathcal{Z}_{0}(\sigma) \subset \operatorname{Spec}\left(\mathbb{C} \llbracket x, y \rrbracket / \mathfrak{m}^{N}\right),
$$


where $\mathfrak{m}=(x, y)$ is the maximal ideal of the origin. Equivalently,

$$
\mathfrak{m}^{N} \subset(A, B) \subset \mathbb{C} \llbracket x, y \rrbracket .
$$

Consider the polynomial 1 -form

$$
\begin{aligned}
\sigma_{\leq N} & =A_{\leq N} d x+B_{\leq N} d y \\
& =\left(A_{d}+A_{d+1}+\cdots+A_{N}\right) d x+\left(B_{d}+B_{d+1}+\cdots+B_{N}\right) d y .
\end{aligned}
$$

While $\sigma_{\leq N}$ need not be almost closed on all of $\mathbb{C}^{2}$ (issues may arise at the zeros of $\sigma$ away from the origin), $\sigma_{\leq N}$ is almost closed in a Zariski open neighbourhood of the origin.

LEMMA 2. In the localised ring $\mathbb{C}[x, y]_{(x, y)}$, the 1-form $\sigma_{\leq N}$ is almost closed,

$$
\left(d \sigma_{\leq N}\right) \subset\left(A_{\leq N}, B_{\leq N}\right) \subset \mathbb{C}[x, y]_{(x, y)} .
$$

Proof. First, we claim $\sigma_{\leq N}$ is almost closed when considered in the ring $\mathbb{C} \llbracket x, y \rrbracket$ of formal power series at the origin of $\mathbb{C}^{2}$. In fact, $d \sigma_{\leq N}$ is

$$
\begin{aligned}
A_{\leq N, y}-B_{\leq N, x} & =\left(A_{y}-B_{x}\right)_{\leq N-1} \\
& =(C A+D B)_{\leq N-1} \\
& =C A_{\leq N}+D B_{\leq N}+\epsilon(N),
\end{aligned}
$$

where $\epsilon(N)$ consists only of terms of degree $\geq N$ and therefore

$$
\epsilon(N) \in \mathfrak{m}^{N} \subset(A, B)
$$

by (10). So we see $\sigma_{\leq N}$ is almost closed modulo $\mathfrak{m}^{N}$, and we can write

$$
\begin{aligned}
\epsilon(N) & =C^{0} A+D^{0} B \\
& =C^{0} A_{\leq N}+D^{0} B_{\leq N}+\left(C^{0} A_{>N}+D^{0} B_{>N}\right) \\
& =C^{0} A_{\leq N}+D^{0} B_{\leq N}+\epsilon(N+1)
\end{aligned}
$$

for series $C^{0}, D^{0} \in \mathbb{C} \llbracket x, y \rrbracket$. Here

$$
\epsilon(N+1) \in \mathfrak{m}^{N+1}=\mathfrak{m} \cdot \mathfrak{m}^{N} \subset \mathfrak{m} \cdot(A, B),
$$

which can therefore be written as

$$
\epsilon(N+1)=C^{1} A_{\leq N}+D^{1} B_{\leq N}+\epsilon(N+2)
$$

just as in (12), with $C^{1}, D^{1} \in \mathfrak{m}$. Continuing inductively, we write

$$
\mathfrak{m}^{k} .(A, B) \supset \mathfrak{m}^{N+k} \ni \epsilon(N+k)=C^{k} A_{\leq N}+D^{k} B_{\leq N}+\epsilon(N+k+1),
$$

where $C^{k}, D^{k} \in \mathfrak{m}^{k}$ consist only of terms of degree $\geq k$. We finally obtain

$$
A_{\leq N, y}-B_{\leq N, x}=\bar{C} A_{\leq N}+\bar{D} B_{\leq N},
$$


where the series

$$
\bar{C}=C+C^{0}+C^{1}+\cdots, \quad \bar{D}=D+D^{0}+D^{1}+\cdots
$$

are convergent in $\mathbb{C} \llbracket x, y \rrbracket$. So $\sigma_{\leq N}$ is indeed almost closed in $\mathbb{C} \llbracket x, y \rrbracket$.

The above results can be written as the vanishing

$$
\left[A_{\leq N, y}-B_{\leq N, x}\right]=0 \quad \text { in the } \mathbb{C} \llbracket x, y \rrbracket \text {-module } \frac{\mathbb{C} \llbracket x, y \rrbracket}{\left(A_{\leq N}, B_{\leq N}\right)},
$$

or equivalently that

$$
0 \longrightarrow \mathbb{C} \llbracket x, y \rrbracket \stackrel{1}{\longrightarrow} \mathbb{C} \llbracket x, y \rrbracket \frac{A_{\leq N, y}-B_{\leq N, x}}{\longrightarrow} \frac{\mathbb{C} \llbracket x, y \rrbracket}{\left(A_{\leq N}, B_{\leq N}\right)}
$$

is exact. We are left with showing similarly that

$$
0 \longrightarrow \mathbb{C}[x, y]_{(x, y)} \stackrel{1}{\longrightarrow} \mathbb{C}[x, y]_{(x, y)} \stackrel{A_{\leq N, y}-B_{\leq N, x}}{\longrightarrow} \frac{\mathbb{C}[x, y](x, y)}{\left(A_{\leq N}, B_{\leq N}\right)}
$$

is also exact.

Now (15) pulls back to (14) via the inclusion

$$
\mathbb{C}[x, y]_{(x, y)} \hookrightarrow \mathbb{C} \llbracket x, y \rrbracket .
$$

Since (16) is flat [4, Theorem 8.8] and a local map of local rings, it is faithfully flat by [4, Theorem 7.2]. Thus, the exactness of (15) follows from that of (14).

5. Non-existence of a potential. Consider again formal power series solutions $\sigma$ of (3) starting at degree $d$. We will show for almost every choice ${ }^{2}$ of $C$ and $D$, the zero scheme $\mathcal{Z}(\sigma)$ is not the critical locus of at the origin of any formal function if $A$ and $B$ are chosen to be general solutions of (7).

THEOREM 3. If $C_{1, x}+D_{1, y} \neq 0$, then for $d \geq 18$ and general choices of $A, B$ satisfying (7), there is no potential function $\Phi \in \mathbb{C} \llbracket x, y \rrbracket$ satisfying

$$
\left(\Phi_{x}, \Phi_{y}\right)=(A, B) \subset \mathbb{C} \llbracket x, y \rrbracket .
$$

Assume a potential function $\Phi \in \mathbb{C} \llbracket x, y \rrbracket$ exists satisfying

$$
\left(\Phi_{x}, \Phi_{y}\right)=(A, B) \subset \mathbb{C} \llbracket x, y \rrbracket .
$$

Then both $\Phi_{x}-A$ and $\Phi_{y}-B$ are in the ideal $(A, B)$, so we have

$$
\begin{aligned}
& \Phi_{x}-A=X A+Y B, \\
& \Phi_{y}-B=Z A+W B,
\end{aligned}
$$

\footnotetext{
${ }^{2}$ In fact, we can set $C=x, D=0$ and $B_{\geq(d+2)}=0$ and the proof still works. But the restriction gives no significant simplification in notation.
} 
for series $X, Y, Z, W \in \mathbb{C} \llbracket x, y \rrbracket$. As usual, we write

$$
X=X_{0}+X_{1}+\cdots
$$

with $X_{k}$ homogeneous of degree $k$ (and similarly for $Y, Z$ and $W$ ). To find $\Phi$ satisfying ${ }^{3}$ (17), integrability

$$
\Phi_{x y}=\Phi_{y x}
$$

is a necessary and sufficient condition. In other words, we must have

$$
-A_{y}+B_{x}=(X A+Y B)_{y}-(Z A+W B)_{x} .
$$

We will analyse equation (18) for $X, Y, Z, W$ order by order modulo higher and higher powers $\mathfrak{m}^{k}$ of the maximal ideal. At each order, the issue is linear. For the first few orders, equation (18) can be easily solved (with several degrees of freedom). But each further stage imposes more stringent conditions on the choices at the previous stage. In degree $d+2$, we will see the conditions become overdetermined, with no non-trivial solutions $X, Y, Z, W$ for general $A, B$ satisfying (3).

Degree d -1 . In homogeneous degree $d-1$, (18) yields

$$
-A_{d, y}+B_{d, x}=X_{0} A_{d, y}+Y_{0} B_{d, y}-Z_{0} A_{d, x}-W_{0} B_{d, x} .
$$

From (4), we have the relation $A_{d, y}=B_{d, x}$. Otherwise the partial derivatives of $A_{d}$ and $B_{d}$ are completely general. Therefore, the resulting equation

$$
0=\left(X_{0}-W_{0}\right) A_{d, y}+Y_{0} B_{d, y}-Z_{0} A_{d, x}
$$

implies the vanishing of $\left(X_{0}-W_{0}\right), Y_{0}$, and $Z_{0}$. Thus, in homogeneous degree $d$, equations (17) become

$$
\begin{aligned}
& \Phi_{d+1, x}=\left(1+X_{0}\right) A_{d}, \\
& \Phi_{d+1, y}=\left(1+X_{0}\right) B_{d},
\end{aligned}
$$

with $\Phi$ having no terms of degree $\leq d$. Since we require $\left(\Phi_{x}, \Phi_{y}\right)$ to generate the ideal $(A, B)$, and since

$$
A_{d}, B_{d} \neq 0
$$

by generality, we find $1+X_{0} \neq 0$. Rescaling $\Phi$, we can assume

$$
1=1+X_{0}
$$

without loss of generality. So we have found the following conditions at order $d-1$ :

$$
X_{0}=Y_{0}=Z_{0}=W_{0}=0 .
$$

\footnotetext{
$\overline{{ }^{3} \text { Equations }}(17)$ imply that only $\left(\Phi_{x}, \Phi_{y}\right) \subset(A, B)$ and has the trivial solution

$$
X=1=W \text { and } Y=0=Z
$$

corresponding to constant $f$. We will rule out the trivial solution by requiring $\left(\Phi_{x}, \Phi_{y}\right)=(A, B)$ shortly.
} 
Degree d. In homogeneous degree $d$, (18) yields

$$
\begin{aligned}
-A_{d+1, y}+B_{d+1, x}= & \left(X_{1} A_{d}\right)_{y}+\left(Y_{1} B_{d}\right)_{y}-\left(Z_{1} A_{d}\right)_{x}-\left(W_{1} B_{d}\right)_{x} \\
& +\left(X_{0} A_{d+1}\right)_{y}+\left(Y_{0} B_{d+1}\right)_{y}-\left(Z_{0} A_{d+1}\right)_{x}-\left(W_{0} B_{d+1}\right)_{x} .
\end{aligned}
$$

By our work in the previous degree (19), the second line on the right vanishes identically. After substituting (6) on the left side, we find

$$
-C_{0} A_{d}-D_{0} B_{d}=\left(X_{1} A_{d}\right)_{y}+\left(Y_{1} B_{d}\right)_{y}-\left(Z_{1} A_{d}\right)_{x}-\left(W_{1} B_{d}\right)_{x}
$$

We rewrite (20) as the vanishing of

$$
\begin{aligned}
& \left(X_{1, y}-Z_{1, x}+C_{0}\right) A_{d}+X_{1} A_{d, y}-Z_{1} A_{d, x} \\
& \quad+\left(Y_{1, y}-W_{1, x}+D_{0}\right) B_{d}+Y_{1} B_{d, y}-W_{1} B_{d, x} .
\end{aligned}
$$

After expanding the linear unknowns out fully via

$$
\begin{aligned}
& X_{1}=X_{1, x} x+X_{1, y} y, \quad Y_{1}=Y_{1, x} x+Y_{1, y} y, \\
& Z_{1}=Z_{1, x} x+Z_{1, y} y, \quad W_{1}=W_{1, x} x+W_{1, y} y,
\end{aligned}
$$

we obtain a relation among the degree $d$ homogeneous polynomials

$$
\begin{array}{r}
A_{d}, x A_{d, x}, x A_{d, y}, y A_{d, x}, y A_{d, y}, \\
B_{d}, x B_{d, x}, x B_{d, y}, y B_{d, x}, y B_{d, y} .
\end{array}
$$

Since $A_{d}$ and $B_{d}$ were chosen generically subject to $A_{d, y}=B_{d, x}$, the polynomials (21) are linearly independent except for the relations

$$
\begin{aligned}
A_{d, y} & =B_{d, x} \quad(\text { multiplied by } x, y), \\
x A_{d, x}+y B_{d, y} & =d A_{d}, \\
x A_{d, x}+y B_{d, y} & =d B_{d} .
\end{aligned}
$$

The first is (4). The last two are the Euler homogeneity relations. A simple check shows that $d \geq 5$ is sufficient to achieve the independence.

Using the first equation of (22) to eliminate $x B_{d, x}$ and $y B_{d, x}$ and the last two to eliminate $A_{d}, B_{d}$, we find the following equations:

$$
\begin{aligned}
& \text { Coefficient of } x A_{d, x}: \quad 0=\left(X_{1, y}-Z_{1, x}+C_{0}\right) / d-Z_{1, x} \text {, } \\
& y A_{d, x}: \quad 0=-Z_{1, y}, \\
& x A_{d, y}: \quad 0=X_{1, x}+\left(Y_{1, y}-W_{1, x}+D_{0}\right) / d-W_{1, x}, \\
& y A_{d, y}: \quad 0=\left(X_{1, y}-Z_{1, x}+C_{0}\right) / d+X_{1, y}-W_{1, y}, \\
& x B_{d, y}: \quad 0=Y_{1, x} \text {, } \\
& y B_{d, y}: \quad 0=\left(Y_{1, y}-W_{1, x}+D_{0}\right) / d+Y_{1, y} \text {. }
\end{aligned}
$$


Therefore, we find

$$
\begin{aligned}
Y_{1, x} & =0=Z_{1, y}, \\
W_{1, y} & =X_{1, y}+Z_{1, x}, \\
X_{1, x} & =Y_{1, y}+W_{1, x}, \\
C_{0} & =(d+1) Z_{1, x}-X_{1, y}, \\
D_{0} & =W_{1, x}-(d+1) Y_{1, y} .
\end{aligned}
$$

Equations (23) can be solved with room to spare - there is a two-dimensional affine space of solutions. However, the constraints in next degree will impose further conditions which specify $X_{1}, Y_{1}, Z_{1}, W_{1}$ uniquely.

Degree d +1 . In homogeneous degree $d+1$, equation (18) yields

$$
\begin{aligned}
-A_{d+2, y}+B_{d+2, x}= & \left(X_{2} A_{d}\right)_{y}+\left(Y_{2} B_{d}\right)_{y}-\left(Z_{2} A_{d}\right)_{x}-\left(W_{2} B_{d}\right)_{x} \\
& +\left(X_{1} A_{d+1}\right)_{y}+\left(Y_{1} B_{d+1}\right)_{y}-\left(Z_{1} A_{d+1}\right)_{x}-\left(W_{1} B_{d+1}\right)_{x} \\
& +\left(X_{0} A_{d+2}\right)_{y}+\left(Y_{0} B_{d+2}\right)_{y}-\left(Z_{0} A_{d+2}\right)_{x}-\left(W_{0} B_{d+2}\right)_{x} .
\end{aligned}
$$

By the constraints (19), the third line vanishes identically. Substituting equation (7) for $k=d+2$ on the left side yields

$$
\begin{aligned}
& -C_{0} A_{d+1}-D_{0} B_{d+1}-C_{1} A_{d}-D_{1} B_{d}=\left(X_{2} A_{d}\right)_{y}+\left(Y_{2} B_{d}\right)_{y}-\left(Z_{2} A_{d}\right)_{x}-\left(W_{2} B_{d}\right)_{x} \\
& +\left(X_{1} A_{d+1}\right)_{y}+\left(Y_{1} B_{d+1}\right)_{y}-\left(Z_{1} A_{d+1}\right)_{x}-\left(W_{1} B_{d+1}\right)_{x}
\end{aligned}
$$

We work first modulo those degree $d+1$ polynomials generated by $A_{d}, B_{d}$ and their first partial derivatives. More preciely, let

$$
V \subset \operatorname{Sym}^{d+1}\left(\mathbb{C}^{2}\right)^{*}
$$

denote the subspace spanned by $x$ and $y$ multiplied by $A_{d}, B_{d}$, and $x^{2}, x y, y^{2}$ multiplied by $A_{d, x}, A_{d, y}, B_{d, x}, B_{d, y}$. The subspace $V$ has dimension 9 due to the relations (22). In the quotient space $\operatorname{Sym}^{d+1}\left(\mathbb{C}^{2}\right)^{*} / V$, equation (24) is

$$
-C_{0} A_{d+1}-D_{0} B_{d+1}=\left(X_{1} A_{d+1}\right)_{y}+\left(Y_{1} B_{d+1}\right)_{y}-\left(Z_{1} A_{d+1}\right)_{x}-\left(W_{1} B_{d+1}\right)_{x},
$$

where all terms are taken $\bmod V$.

Equation (25) has form identical to equation (20) analysed in the previous degree with $A_{d}, B_{d}$ replaced by $A_{d+1}, B_{d+1}$. The analysis of the previous sections applies again here: The first relation of (22) holds mod $V$ by (6), and the two Euler relations hold with $d$ replaced by $d+1$. By generality, for $d \geq 13$, the polynomials $A_{d+1}, B_{d+1}$ and their partial derivatives (multiplied by $x, y$ ) are independent of $A_{d}, B_{d}$ (multiplied by $x, y)$ and their partial derivatives (multiplied by $x^{2}, x y, y^{2}$ ) except for relation (6) and the Euler relations. We conclude the equations corresponding to (23) hold (with $d$ 
replaced by $d+1)$ :

$$
\begin{aligned}
Y_{1, x} & =0=Z_{1, y}, \\
W_{1, y} & =X_{1, y}+Z_{1, x}, \\
X_{1, x} & =Y_{1, y}+W_{1, x}, \\
C_{0} & =(d+2) Z_{1, x}-X_{1, y}, \\
D_{0} & =W_{1, x}-(d+2) Y_{1, y} .
\end{aligned}
$$

After combining with the original equations (23), we find

$$
\begin{aligned}
& Y_{1, x}=0=Y_{1, y}, \\
& Z_{1, x}=0=Z_{1, y}, \\
& X_{1, y}=W_{1, y}=-C_{0}, \\
& X_{1, x}=W_{1, x}=D_{0} .
\end{aligned}
$$

We have uniquely solved for $X_{1}, Y_{1}, Z_{1}, W_{1}$.

We next consider $X_{2}, Y_{2}, Z_{2}, W_{2}$. There will be no obstruction to solving for $X_{2}, Y_{2}, Z_{2}, W_{2}$ here. However, in the next degree, we will find further constraints: the resulting overdetermined system for $X_{2}, Y_{2}, Z_{2}, W_{2}$ will have solutions only if $C_{1, x}+D_{1, y}=0$.

After substituting constraints (26) into (24), we obtained a simpler equation:

$$
-C_{1} A_{d}-D_{1} B_{d}=\left(X_{2} A_{d}\right)_{y}+\left(Y_{2} B_{d}\right)_{y}-\left(Z_{2} A_{d}\right)_{x}-\left(W_{2} B_{d}\right)_{x}+X_{1}\left(A_{d+1, y}-B_{d+1, x}\right) .
$$

By (6) and (26), the last term $X_{1}\left(A_{d+1, y}-B_{d+1, x}\right)$ is

$$
X_{1}\left(C_{0} A_{d}+D_{0} B_{d}\right)=\left(D_{0} x-C_{0} y\right)\left(C_{0} A_{d}+D_{0} B_{d}\right) .
$$

We define new terms

$$
\widetilde{C}_{1}=C_{1}+C_{0}\left(D_{0} x-C_{0} y\right), \quad \widetilde{D}_{1}=D_{1}+D_{0}\left(D_{0} x-C_{0} y\right) .
$$

Then we can write equation (24) as

$$
-\widetilde{C}_{1} A_{d}-\widetilde{D}_{1} B_{d}=\left(X_{2} A_{d}\right)_{y}+\left(Y_{2} B_{d}\right)_{y}-\left(Z_{2} A_{d}\right)_{x}-\left(W_{2} B_{d}\right)_{x} .
$$

Note the similarity to (20).

We solve (27) following our approach to (20). After expanding the unknowns,

$$
\begin{aligned}
X_{2} & =X_{2, x x} \frac{x^{2}}{2}+X_{2, x y} x y+X_{2, y y} \frac{y^{2}}{2}, \\
Y_{2} & =Y_{2, x x} \frac{x^{2}}{2}+Y_{2, x y} x y+Y_{2, y y} \frac{y^{2}}{2}, \\
Z_{2} & =Z_{2, x x} \frac{x^{2}}{2}+Z_{2, x y} x y+Z_{2, y y} \frac{y^{2}}{2}, \\
W_{2} & =W_{2, x x} \frac{x^{2}}{2}+W_{2, x y} x y+W_{2, y y} \frac{y^{2}}{2},
\end{aligned}
$$


we obtain

$$
\begin{aligned}
-\left(\tilde{C}_{1, x} x+\tilde{C}_{1, y} y\right) A_{d}-\left(\tilde{D}_{1, x} x+\tilde{D}_{1, y} y\right) B_{d} \\
=\left(X_{2, x x} \frac{x^{2}}{2}+X_{2, x y} x y+X_{2, y y} \frac{y^{2}}{2}\right) A_{d, y}+\left(X_{2, x y} x+X_{2, y y} y\right) A_{d} \\
\quad+\left(Y_{2, x x} \frac{x^{2}}{2}+Y_{2, x y} x y+Y_{2, y y} \frac{y^{2}}{2}\right) B_{d, y}+\left(Y_{2, x y} x+Y_{2, y y} y\right) B_{d} \\
-\left(Z_{2, x x} \frac{x^{2}}{2}+Z_{2, x y} x y+Z_{2, y y} \frac{y^{2}}{2}\right) A_{d, x}-\left(Z_{2, x x} x+Z_{2, x y} y\right) A_{d} \\
-\left(W_{2, x x} \frac{x^{2}}{2}+W_{2, x y} x y+W_{2, y y} \frac{y^{2}}{2}\right) B_{d, x}-\left(W_{2, x x} x+W_{2, x y} y\right) B_{d} .
\end{aligned}
$$

We consider the above to be a relation among

$$
\begin{array}{r}
x A_{d}, y A_{d}, x^{2} A_{d, x}, x y A_{d, x}, y^{2} A_{d, x}, x^{2} A_{d, y}, x y A_{d, y}, y^{2} A_{d, y}, \\
x B_{d}, y B_{d}, x^{2} B_{d, x}, x y B_{d, x}, y^{2} B_{d, x}, x^{2} B_{d, y}, x y B_{d, y}, y^{2} B_{d, y} .
\end{array}
$$

By generality, for $d \geq 7$, these are linearly independent degree $d+1$ homogeneous polynomials modulo (4) and the Euler relations (22):

$$
\begin{array}{rlrl}
A_{d, y}=B_{d, x} & & \left(\text { multiplied by } x^{2}, x y, y^{2}\right) \\
x A_{d, x}+y A_{d, y}=d A_{d} & & \text { (multiplied by } x, y) \\
x B_{d, x}+y B_{d, y} & =d B_{d} & & \text { (multiplied by } x, y)
\end{array}
$$

We use (29) to eliminate the terms

$$
x^{2} B_{d, x}, x y B_{d, x}, y^{2} B_{d, x}, x A_{d}, y A_{d}, x B_{d}, y B_{d}
$$

on the right-hand side of (28). Then independence yields the following equations:

$$
\begin{aligned}
x^{2} A_{d, x}: & 0=\widetilde{C}_{1, x} / d-Z_{2, x x} / 2+X_{2, x y} / d-Z_{2, x x} / d, \\
x y A_{d, x}: & 0=\widetilde{C}_{1, y} / d-Z_{2, x y}+X_{2, y y} / d-Z_{2, x y} / d, \\
y^{2} A_{d, x}: & 0=-Z_{2, y y} / 2, \\
x^{2} A_{d, y}: & 0=\widetilde{D}_{1, x} / d+X_{2, x x} / 2-W_{2, x x} / 2+Y_{2, x y} / d-W_{2, x x} / d, \\
x y A_{d, y}: & 0=\widetilde{C}_{1, x} / d+\widetilde{D}_{1, y} / d+X_{2, x y}-W_{2, x y}+X_{2, x y} / d \\
& \quad-Z_{2, x x} / d+Y_{2, y y} / d-W_{2, x y} / d, \\
y^{2} A_{d, y}: & 0=\widetilde{C}_{1, y} / d+X_{2, y y} / 2-W_{2, y y} / 2+X_{2, y y} / d-Z_{2, x y} / d, \\
x^{2} B_{d, y}: & 0=Y_{2, x x} / 2, \\
x y B_{d, y}: & 0=\widetilde{D}_{1, x} / d+Y_{2, x y}+Y_{2, x y} / d-W_{2, x x} / d, \\
y^{2} B_{d, y}: & 0=\widetilde{D}_{1, y} / d+Y_{2, y y} / 2+Y_{2, y y} / d-W_{2, x y} / d .
\end{aligned}
$$

Use the second equation to eliminate $X_{2, y y}$ from the sixth equation. Use the eighth equation to eliminate $W_{2, x x}$ from the fourth equation. Finally, use the first and last equations to remove $X_{2, x y}$ and $W_{2, x y}$ from the fifth one. Tidying up, we find $Z_{2, y y}=$ 
$0=Y_{2, x x}$ and

$$
\begin{aligned}
(d+2) Z_{2, x x}-2 X_{2, x y} & =2 \widetilde{C}_{1, x}, \\
(d+1) Z_{2, x y}-X_{2, y y} & =\widetilde{C}_{1, y}, \\
X_{2, x x}-(d+3) Y_{2, x y} & =\widetilde{D}_{1, x}, \\
(d+3)\left(Z_{2, x x}-Y_{2, y y}\right) & =2\left(\widetilde{C}_{1, x}+\widetilde{D}_{1, y}\right), \\
-W_{2, y y}+(d+3) Z_{2, x y} & =\widetilde{C}_{1, y}, \\
(d+1) Y_{2, x y}-W_{2, x x} & =-\widetilde{D}_{1, x}, \\
(d+2) Y_{2, y y}-2 W_{2, x y} & =-2 \widetilde{D}_{1, y} .
\end{aligned}
$$

Therefore, we can specify that $Z_{2, x x}, Z_{2, x y}$ and $Y_{2 x y}$ arbitrarily and uniquely solve for $X_{2}, Y_{2}, Z_{2}, W_{2}$ after that. However, the conditions at the next degree will place further constraints.

Degree $\mathbf{d}+2$. In homogeneous degree $d+2$, equation (18) yields

$$
\begin{aligned}
-A_{d+3, y}+B_{d+3, x}= & \left(X_{3} A_{d}\right)_{y}+\left(Y_{3} B_{d}\right)_{y}-\left(Z_{3} A_{d}\right)_{x}-\left(W_{3} B_{d}\right)_{x} \\
& +\left(X_{2} A_{d+1}\right)_{y}+\left(Y_{2} B_{d+1}\right)_{y}-\left(Z_{2} A_{d+1}\right)_{x}-\left(W_{2} B_{d+1}\right)_{x} \\
& +\left(X_{1} A_{d+2}\right)_{y}+\left(Y_{1} B_{d+2}\right)_{y}-\left(Z_{1} A_{d+2}\right)_{x}-\left(W_{1} B_{d+2}\right)_{x} \\
& +\left(X_{0} A_{d+3}\right)_{y}+\left(Y_{0} B_{d+3}\right)_{y}-\left(Z_{0} A_{d+3}\right)_{x}-\left(W_{0} B_{d+3}\right)_{x} .
\end{aligned}
$$

By constraints (19), the fourth line vanishes identically. Substituting (7) for $k=d+3$ on the left-hand side yields

$$
-C_{0} A_{d+2}-D_{0} B_{d+2}-C_{1} A_{d+1}-D_{1} B_{d+1}-C_{2} A_{d}-D_{2} B_{d} .
$$

Work modulo the (12-dimensional space of) degree $d+2$ homogeneous polynomials generated by $A_{d}, B_{d}$ (multiplied by $x^{2}, x y$ or $y^{2}$ ) and their first partial derivatives (multiplied by $\left.x^{3}, x^{2} y, x y^{2}, y^{3}\right)$. We find

$$
\begin{aligned}
& -C_{0} A_{d+2}-D_{0} B_{d+2}-C_{1} A_{d+1}-D_{1} B_{d+1}=\left(X_{2} A_{d+1}\right)_{y}+\left(Y_{2} B_{d+1}\right)_{y} \\
& -\left(Z_{2} A_{d+1}\right)_{x}-\left(W_{2} B_{d+1}\right)_{x}+\left(X_{1} A_{d+2}\right)_{y}+\left(Y_{1} B_{d+2}\right)_{y}-\left(Z_{1} A_{d+2}\right)_{x}-\left(W_{1} B_{d+2}\right)_{x} .
\end{aligned}
$$

Substituting (26) for $X_{1}, Y_{1}, Z_{1}, W_{1}$ into the third line and moving it to the first gives

$$
\begin{aligned}
& -C_{1} A_{d+1}-D_{1} B_{d+1}-\left(D_{0} x-C_{0} y\right)\left(A_{d+2, y}-B_{d+2, x}\right) \\
& =\left(X_{2} A_{d+1}\right)_{y}+\left(Y_{2} B_{d+1}\right)_{y}-\left(Z_{2} A_{d+1}\right)_{x}-\left(W_{2} B_{d+1}\right)_{x} .
\end{aligned}
$$

By (7) for $k=d+2$, we know the term

$$
A_{d+2, y}-B_{d+2, x}=C_{0} A_{d+1}+D_{0} B_{d+1}+C_{1} A_{d}+D_{1} B_{d} .
$$

Since we are working modulo $A_{d}, B_{d}$, we obtain

$$
-\widetilde{C}_{1} A_{d+1}-\widetilde{D}_{1} B_{d+1}=\left(X_{2} A_{d+1}\right)_{y}+\left(Y_{2} B_{d+1}\right)_{y}-\left(Z_{2} A_{d+1}\right)_{x}-\left(W_{2} B_{d+1}\right)_{x},
$$

where

$$
\widetilde{C}_{1}=C_{1}+C_{0}\left(D_{0} x-C_{0} y\right), \quad \widetilde{D}_{1}=D_{1}+D_{0}\left(D_{0} x-C_{0} y\right)
$$

as before. 
Equation (31) is precisely the same as equation (27) studied at the previous degree with $d$ replaced by $d+1$. Also, relations (29) still hold (after replacing $d$ by $d+1$ ) by (6), since we are working mod $A_{d}, B_{d}$, and these are the only relations which hold for general $A_{d+1}, B_{d+1}$ once $d \geq 18$.

Hence, the analysis in the previous degree applies here verbatim. We derive the same equations (30) with $d$ replaced by $d+1$. The first is $Z_{2, y y}=0=Y_{2, x x}$. And the rest are

$$
\begin{aligned}
(d+3) Z_{2, x x}-2 X_{2, x y} & =2 \widetilde{C}_{1, x}, \\
(d+2) Z_{2, x y}-X_{2, y y} & =\widetilde{C}_{1, y}, \\
X_{2, x x}-(d+4) Y_{2, x y} & =\widetilde{D}_{1, x}, \\
(d+4)\left(Z_{2, x x}-Y_{2, y y}\right) & =2\left(\widetilde{C}_{1, x}+\widetilde{D}_{1, y}\right), \\
-W_{2, y y}+(d+4) Z_{2, x y} & =\widetilde{C}_{1, y}, \\
(d+2) Y_{2, x y}-W_{2, x x} & =-\widetilde{D}_{1, x}, \\
(d+3) Y_{2, y y}-2 W_{2, x y} & =-2 \widetilde{D}_{1, y} .
\end{aligned}
$$

From the central equation and the counterpart in (30), we obtain a necessary condition for there to exist any solutions:

$$
\widetilde{C}_{1, x}+\widetilde{D}_{1, y}=0
$$

or equivalently,

$$
C_{1, x}+D_{1, y}=0 .
$$

We have completed the proof of Theorem 3.

Since $C_{1}$ and $D_{1}$ can be chosen arbitrarily at the beginning, choosing them to violate (32) will imply the non-existence of a potential $\Phi$. More precisely, when $A_{k}, B_{k}$ are general solutions of equations (7), the resulting almost closed 1-form generates an ideal at the origin which is not the critical locus of any formal power series $\Phi \in \mathbb{C} \llbracket x, y \rrbracket$.

By Lemma 2, we can find an algebraic almost closed 1-form on a Zariski open set of the origin in $\mathbb{C}^{2}$ which is not the critical locus of any holomorphic (or even formal) function $\Phi$ defined near the origin of $\mathbb{C}^{2}$.

6. Embedding in higher dimensions. Let $\sigma$ be an algebraic almost closed 1-form on a Zariski open set of the origin in $\mathbb{C}^{2}$ (as constructed above) whose zero locus $\mathcal{Z}(\sigma)$ is both 0 -dimensional and not the critical locus of any holomorphic potential function $\Phi$ near the origin in $\mathbb{C}^{2}$.

Proposition 4. The scheme $\mathcal{Z}(\sigma) \subset \mathbb{C}^{2}$ cannot be written as the critical locus $\mathcal{Z}(d \Phi)$ of any holomorphic function $\Phi$ on a non-singular variety.

Proof. For contradiction, suppose

$$
\mathcal{Z}(\sigma)=\mathcal{Z}(d \Phi)
$$

for some holomorphic function $\Phi$ on a non-singular analytic variety $A$. Let $\mathcal{Z}$ denote $\mathcal{Z}(\sigma)=\mathcal{Z}(d \Phi)$. 
We show $A$ can be cut down to two dimensions. Since $\mathcal{Z}$ has a two-dimensional Zariski tangent space, $A$ has a product structure (perhaps after shrinking to an Euclidean open neighbourhood),

$$
A=B \times C
$$

with $B$ non-singular of dimension 2 and

$$
\mathcal{Z} \subset B \times\{c\} \subset B \times C=A .
$$

Let $b \in B$ be the point at which $\mathcal{Z}$ is supported, and

$$
p=(b, c) \in A \text {. }
$$

Now $\left.D(d \Phi)\right|_{p}$ is injective on $T_{c} C$ because $T_{c} C$ is a complement to the kernel $T_{b} B$. Since $\left.D(d \Phi)\right|_{p}$ is symmetric, $\left.\operatorname{Im} D(d \Phi)\right|_{p}$ lies in the annihilator of $\left.\operatorname{ker} D(d \Phi)\right|_{p}$, which is $\left.\Omega_{C}\right|_{c}$. Thus, the composition

$$
T_{C} \longrightarrow T_{A} \stackrel{D(d \Phi)}{\longrightarrow} \Omega_{A} \longrightarrow \Omega_{C}
$$

on $\mathcal{Z}$ is injective at $p$. The composition is therefore an isomorphism at $p$, and hence, by openness, an isomorphism in a neighbourhood of $\mathcal{Z}$.

Thus, writing $\left(d_{B} \Phi, d_{C} \Phi\right)$ for $d \Phi=\left(d_{B \times C / C} \Phi, d_{B \times C / B} \Phi\right)$ in the product structure (33), we find that the zero locus

$$
B^{\prime}=\mathcal{Z}\left(d_{C} \Phi\right)
$$

of $d_{C} \Phi$ is tangent to $B$ at $p$ and smooth and two-dimensional in a neighbourhood. Shrinking if necessary, we can assume that $B^{\prime}$ is everywhere non-singular and never tangent to the $C$ fibres of $A=B \times C$.

Now $\mathcal{Z}$ is the zero locus of $d_{B} \Phi$ on $B^{\prime}$. By the tangency condition, $\mathcal{Z}$ is the same as the zero locus of $\left.(d \Phi)\right|_{B^{\prime}}=d\left(\left.\Phi\right|_{B^{\prime}}\right)$. Thus, $\mathcal{Z}$ is the critical locus of $\left.\Phi\right|_{B^{\prime}}$, with $B^{\prime}$ non-singular and two-dimensional, contradicting Theorem 3.

AcKnowledgements. We would like to thank Dominic Joyce for showing us the potential (2) for the zeros of the 1-form (1) and pointing out the error in [5, Appendix A.5]. Specifically, the averaging argument in Corollary 34 is incorrect. The error there does not affect the other counterexamples in [5, Appendix A].

\section{REFERENCES}

1. K. Behrend, Donaldson-Thomas invariants via microlocal geometry, Ann. Math. 170 (2009) 1307-1338. math.AG/0507523.

2. K. Behrend and B. Fantechi, Symmetric obstruction theories and Hilbert schemes of points on threefolds, Algebra Number Theory 2 (2008) 313-345. math.AG/0512556.

3. C. Brav, V. Bussi and D. Joyce, A Darboux theorem for derived schemes with shifted symplectic structure, (2013) arXiv:1305.6302. 1989).

4. H. Matsumura, Commutative ring theory (Cambridge University Press, Cambridge, UK,

5. D. Maulik, R Pandharipande and R. P. Thomas, Curves on K3 surfaces and modular forms, J. Topol. 3 (2010) 937-996. arXiv:1001.2719v3.

6. T. Pantev, B. Toën, M. Vaquie and G. Vezzosi, Quantization and derived moduli spaces I: shifted symplectic structures, arXiv:1111.3209. 